\title{
GROUND-STATES FOR THE LIQUID DROP AND TFDW MODELS WITH LONG-RANGE ATTRACTION
}

\author{
STAN ALAMA, LIA BRONSARD, RUSTUM CHOKSI, AND IHSAN TOPALOGLU
}

\begin{abstract}
We prove that both the liquid drop model in $\mathbb{R}^{3}$ with an attractive background nucleus and the Thomas-Fermi-Dirac-von Weizsäcker (TFDW) model attain their ground-states for all masses as long as the external potential $V(x)$ in these models is of long range, that is, it decays slower than Newtonian (e.g., $V(x) \gg|x|^{-1}$ for large $|x|$.) For the TFDW model we adapt classical concentration-compactness arguments by Lions, whereas for the liquid drop model with background attraction we utilize a recent compactness result for sets of finite perimeter by Frank and Lieb.
\end{abstract}

\section{INTRODUCTION}

In this note we consider ground-states of two mass-constrained variational problems containing an external attractive potential to the origin which is super-Newtonian at long ranges. The first problem consists of a variant of Gamow's liquid drop problem [8, 10, 23] perturbed by an attractive background potential $V(x)$, with long range decay, in the sense that $V(x) \gg|x|^{-1}$ for large $|x|$. The second problem is a variant of the ThomasFermi-Dirac-von Weizsäcker (TFDW) functional, again subject to an external attractive potential $V(x)$ which is "super-Newtonian".

Let us first state the two problems precisely. The variant of the liquid drop problem is given by

$$
e_{V}(M):=\inf \left\{\mathrm{E}_{V}(u): u \in B V\left(\mathbb{R}^{3} ;\{0,1\}\right), \int_{\mathbb{R}^{3}} u d x=M\right\},
$$

where the energy functional $\mathrm{E}_{V}$ is defined as

$$
\mathrm{E}_{V}(u):=\int_{\mathbb{R}^{3}}|\nabla u|+\int_{\mathbb{R}^{3}} \int_{\mathbb{R}^{3}} \frac{u(x) u(y)}{|x-y|} d x d y-\int_{\mathbb{R}^{3}} V(x) u(x) d x .
$$

Here the first term in $\mathrm{E}_{V}$ computes the total variation of the function $u$, i.e.,

$$
\int_{\mathbb{R}^{3}}|\nabla u|=\sup \left\{\int_{\mathbb{R}^{3}} u \operatorname{div} \phi d x: \phi \in C_{0}^{1}\left(\mathbb{R}^{3} ; \mathbb{R}^{3}\right),|\phi| \leqslant 1\right\}
$$

and is equal to $\operatorname{Per}_{\mathbb{R}^{3}}\left(\left\{x \in \mathbb{R}^{3}: u(x)=1\right\}\right)$ since $u$ takes on only the values 0 and 1 .

The variant of the TFDW problem we consider here is to find

$$
I_{V}(M):=\inf \left\{\mathscr{E}_{V}(u): u \in H^{1}\left(\mathbb{R}^{3}\right), \int_{\mathbb{R}^{3}}|u|^{2} d x=M\right\},
$$

Date: July 6, 2021.

1991 Mathematics Subject Classification. 35Q40, 35Q70, 49Q20, 49S05, 82D10.

Key words and phrases. liquid drop model, background nucleus, Thomas-Fermi-Dirac-von Weizsäcker theory, ground-states, global existence, concentration-compactness method. 
where

$$
\mathscr{E}_{V}(u):=\int_{\mathbb{R}^{3}}\left(|\nabla u|^{2}+|u|^{10 / 3}-|u|^{8 / 3}-V(x)|u|^{2}\right) d x+\frac{1}{2} \int_{\mathbb{R}^{3}} \int_{\mathbb{R}^{3}} \frac{|u(x)|^{2}|u(y)|^{2}}{|x-y|} d x d y .
$$

In the original TFDW problem (see the works of Benguria, Brézis, Lieb [2], Le Bris, Lions [12], and Lieb [13] for detailed surveys on this classical theory), the potential is taken to be

$$
V_{Z}(x):=\frac{Z}{|x|},
$$

simulating an attracting point charge at the origin with charge $Z$. With this physical choice of potential, both the liquid drop and TFDW problems have been shown to exhibit existence for small $M$ and nonexistence for large $M$. In particular, for the liquid drop model it has recently been shown by Lu and Otto, and by Frank, Nam and van den Bosch that

- (nonexistence, Theorem 1.4 of Frank, Nam, van den Bosch [7]) if $E_{V_{Z}}$ has a minimizer, then $M \leqslant \min \left\{2 Z+8, Z+C Z^{1 / 3}+8\right\}$ for some $C>0$; and,

- (existence, Theorem 2 of Lu, Otto [17]) there exists a constant $c>0$ so that for $M \leqslant Z+c$ the unique minimizer of $\mathrm{E}_{V_{Z}}$ is given by the ball $\chi_{B(0, R)}$,

where $R=\left(M / \omega_{3}\right)^{1 / 3}$ and $\omega_{3}$ denotes the volume of the unit ball in $\mathbb{R}^{3}$. Similar (and older) existence results hold for the TFDW problem. The existence of solutions to the classical TFDW problem was established by Lions [15] for $M \leqslant Z$ and extended to $M \leqslant Z+c$ for some constant $c>0$ by Le Bris [11]. The nonexistence of ground-states for large values of $M$ (or small values of $Z$ ) is only recently proved by Frank, Nam and van den Bosch [7,22, In a separate paper Nam and van den Bosch 22 also consider more general external potentials which are short-ranged, i.e., $\lim _{|x| \rightarrow \infty}|x| V(x)=0$. Motivated by their result, here we look at the complementary case, in which the external potential is asymptotically larger than Newtonian at infinity.

These functionals can be viewed as mathematical paradigms for the existence and nonexistence of coherent structures based upon a mass parameter. Since both problems are driven by a repulsive potential of Coulombic (Newtonian) type, it is natural to expect that if the confining external potential $V$ was even slightly stronger (at long ranges) than Newtonian, global existence would be restored for all masses. In this note we prove that this is indeed the case.

For the liquid drop problem $e_{V}$, we consider the external potentials $V$ which satisfy the following hypotheses:

(H1) $V \geqslant 0$, and $V \in L_{\text {loc }}^{1}\left(\mathbb{R}^{3}\right)$.

(H2) $\lim _{t \rightarrow \infty} t\left(\inf _{|x|=t} V(x)\right)=\infty$.

(H3) $\lim _{|x| \rightarrow \infty} V(x)=0$.

On the other hand, to ensure that the energy $\mathscr{E}_{V}$ is bounded below, we assume that $V$ satisfies

$\left(\mathrm{H} 1^{\prime}\right) V \geqslant 0$, and $V \in L^{3 / 2}\left(\mathbb{R}^{3}\right)+L^{\infty}\left(\mathbb{R}^{3}\right)$, 
instead of (H1), along with (H2) and (H3). Hypothesis (H2) implies that these potentials are long-ranged but only slightly more attractive than Newtonian. A typical example of such an external potential is

$$
V(x)=\frac{1}{|x|^{1-\epsilon}}
$$

for $0<\epsilon<1$, or a linear combination of functions of this form. Although these potentials have only slightly longer range than $|x|^{-1}$, this is sufficient to ensure existence of groundstates for the modified liquid drop and TFDW problems, $e_{V}$ and $I_{V}$, for all $M>0$.

Theorem 1 (Liquid drop model). Suppose $V$ satisfies (H1) (H3). Then for any $M>0$ the problem $e_{V}(M)$ given by $(\mathrm{LD})$ has a solution.

Theorem 2 (TFDW model). Suppose $V$ satisfies $\left(\mathrm{H}^{\prime}\right),(\mathrm{H} 2)$, and (H3), Then for any $M>0$ the problem $I_{V}(M)$ given by (TFDW) has a solution.

Remark 3. While we do obtain existence of ground-states for all masses $M$, we do not expect that the attractive potential $V$ stabilizes the single droplet solution $\chi_{B\left(0,\left(M / \omega_{3}\right)\right)^{1 / 3}}$ for large values of $M$. Rather, we expect that mass splitting does indeed occur (as it does for the unperturbed liquid drop problem [9, 10, 18]) but the resulting components are confined by the external potential $V$ and cannot escape to infinity. This expectation is reflected in our approach to the proof of the two theorems above.

While the mathematical motivations for these results are clear, let us now comment on the physicality of the long-range super-Newtonian attraction. For the quantum TFDW model, we do not know of any physical situation which would support an "exterior" potential producing super-Newtonian attraction. However we note that these functionals, in particular the liquid drop energy, can be used as phenomenological models for charged or gravitating masses at all length scales. Consideration of super-Newtonian forces appears in several theories at the cosmological level, and in fact the validity of Newton's law at long distances has been a longstanding interest in physics. As Finzi notes [5], for example, stability of cluster of galaxies implies stronger attractive forces at long distances than that predicted by Newton's law. Motivated by similar observations, Milgrom [20] introduced the modified Newtonian dynamics (MOND) theory which suggests that the gravitational force experienced by a star in the outer regions of a galaxy must be stronger than Newton's law (see also works of Bugg [3], and Milgrom [21] for a survey, and Bekenstein's work [1]).

Outline of the paper: The proofs of Theorems 1 and 2 follow the same basic strategy: to obtain a contradiction, we assume that minimizing sequences lose compactness, and use concentration compactness techniques to show that it is because of the splitting and dispersion of mass to infinity ("dichotomy"). For the liquid drop model, we utilize a recent technical concentration-compactness result for sets of finite perimeter by Frank and Lieb [6] to prove a lower bound on the energy in case minimizing sequences $u_{n}$ lose compactness via splitting, of the form

$$
\lim _{n \rightarrow \infty} \mathrm{E}_{V}\left(u_{n}\right) \geqslant e_{V}\left(m_{0}\right)+e_{0}\left(m_{1}\right)+e_{0}\left(M-m_{0}-m_{1}\right),
$$

where $0<m_{i}<M$ with $m_{0}+m_{1} \leqslant M$. However, thanks to the super-Newtonian decay of $V$ we then show that $e_{V}(M)$ actually lies strictly below the value given in (1.3). This 
is a variant on the original "strict subadditivity" argument introduced by Lions [15] for the classical TFDW model with $V(x)=|x|^{-1}$, and subsequently used in innumerable treatments of variational problems with loss of compactness.

In section 3 we adapt recent arguments by Nam and van den Bosch 22 along with estimates of Lions [15] and Le Bris [1] to prove Theorem 2. Although the variational structure of TFDW is nearly the same as the liquid drop model, the components are not compactly supported, so we require an additional argument to verify that they decay sufficiently rapidly (in fact exponentially) in order to calculate the interaction between components.

\section{Proof of Theorem 1}

Our proof relies on a recent concentration-compactness type result for sets of finite perimeter by Frank and Lieb [6]. While similar compactness results are known and could be adapted here (for example, the classical theory of Lions [16], and results for minimizing clusters which can be found in Chapter 29 of Maggi [19]), the results of Frank and Lieb are particularly well-suited for our purposes. Throughout the proof of Theorem 1, we specifically use Proposition 2.1, and Lemmas 2.2 and 2.3 of Frank and Lieb [6].

As noted in the introduction our goal is to obtain a splitting property (1.3) for $e_{V}(M)$ involving the "minimization problem at infinity" $e_{0}$ given by

$$
e_{0}(M):=\inf \left\{\mathrm{E}_{0}(u): u \in B V\left(\mathbb{R}^{3} ;\{0,1\}\right), \text { and } \int_{\mathbb{R}^{3}} u d x=M\right\},
$$

where

$$
\mathrm{E}_{0}(u):=\int_{\mathbb{R}^{3}}|\nabla u|+\int_{\mathbb{R}^{3}} \int_{\mathbb{R}^{3}} \frac{u(x) u(y)}{|x-y|} d x d y
$$

We will also use the following simple weak compactness result for the confinement term, which is convenient to state in general terms.

Lemma 4. Let $A_{n} \subset \mathbb{R}^{3}$ be a sequence of sets with $\left|A_{n}\right| \leqslant M$ which converge to zero locally, i.e., $\chi_{A_{n}} \rightarrow 0$ in $L_{\mathrm{loc}}^{1}\left(\mathbb{R}^{3}\right)$. Then

$$
\int_{A_{n}} V d x=\int_{\mathbb{R}^{3}} V \chi_{A_{n}} d x \rightarrow 0 \quad \text { as } n \rightarrow \infty .
$$

Proof. By hypothesis (H3) for any $\epsilon>0$, there exists $R>0$ so that if $V_{\infty}:=V \chi_{B_{R}^{c}}$, then $0 \leqslant V_{\infty}<\frac{\epsilon}{3 M}$. By (H1)] on the other hand, we define $V_{1}:=V \chi_{B_{R} \backslash E_{K}}$, where $E_{K}=\left\{x \in B_{R}: 0 \leqslant V(x) \leqslant K\right\}$ and $K$ is chosen with $\left\|V_{1}\right\|_{L^{1}\left(\mathbb{R}^{3}\right)}<\frac{\epsilon}{3}$. Finally, let $V_{2}:=V \chi_{E_{K}}$, which is supported in $B_{R}$, and satisfies $\left\|V_{2}\right\|_{L^{\infty}\left(\mathbb{R}^{3}\right)} \leqslant K$.

Now with these choices we have a decomposition of $V$ into $V_{1}+V_{2}+V_{\infty}$, depending on $\epsilon$ and $K$. Using this decomposition

$$
0 \leqslant \int_{A_{n}} V d x \leqslant\left\|V_{1}\right\|_{L^{1}}+K\left|A_{n} \cap B_{R}\right|+\frac{\epsilon}{3 M}\left|A_{n}\right|<K\left|A_{n} \cap B_{R}\right|+\frac{2 \epsilon}{3}<\epsilon,
$$

for all $n$ large enough, since $\left|A_{n} \cap B_{R}\right| \rightarrow 0$ as $n \rightarrow \infty$ by local convergence of the sets $A_{n}$. 
Proof of Theorem 1. First, by (H1) and (H3) we may write $V=V \chi_{B_{R}}+V \chi_{B_{R}^{c}} \in L^{1}+L^{\infty}$, where $R$ is chosen so that $\left\|V \chi_{B_{R}^{c}}\right\|_{L^{\infty}\left(\mathbb{R}^{3}\right)} \leqslant 1$. Then, for any $u=\chi_{\Omega}$ with $|\Omega|=M$,

$$
\int_{\mathbb{R}^{3}} V u d x \leqslant\|V\|_{L^{1}\left(B_{R}\right)}+M
$$

hence, $e_{V}(M)>-\infty$. Now, let $\left\{u_{n}\right\}_{n \in \mathbb{N}} \subset B V\left(\mathbb{R}^{3} ;\{0,1\}\right)$ with $\int_{\mathbb{R}^{3}} u_{n} d x=M$ be a minimizing sequence for the energy $\mathrm{E}_{V}$, i.e., $\lim _{n \rightarrow \infty} \mathrm{E}_{V}\left(u_{n}\right)=e_{V}(M)$. By the above estimate on the confinement term, the minimizing sequence has uniformly bounded perimeter, $\int_{\mathbb{R}^{3}}\left|\nabla u_{n}\right| \leqslant C$ independent of $n$. Define the sets of finite perimeter $\Omega_{n} \subset \mathbb{R}^{3}$ so that $\chi_{\Omega_{n}}=u_{n}$, and $\left|\Omega_{n}\right|=M$ for all $n \in \mathbb{N}$.

Step 1. First, we set up our contradiction argument. By the compact embedding of $B V\left(\mathbb{R}^{3}\right)$ in $L_{\text {loc }}^{1}\left(\mathbb{R}^{3}\right)$ (see e.g. Corollary 12.27 in Maggi [19]) there exists a subsequence and a set of finite perimeter $\Omega^{0} \subset \mathbb{R}^{3}$ so that $\Omega_{n} \rightarrow \Omega^{0}$ locally, that is, $u_{n} \rightarrow \chi_{\Omega^{0}}:=w^{0}$ in $L_{\text {loc }}^{1}\left(\mathbb{R}^{3}\right)$. At this point, we admit the possibility that $w^{0} \equiv 0$, i.e., $\left|\Omega^{0}\right|=0$. However, in Step 4 we show that $w^{0} \not \equiv 0$.

If the limit set $\left|\Omega^{0}\right|=M$, then we are done. Indeed, since $\left\{u_{n}\right\}_{n \in \mathbb{N}}$ is locally convergent in $L^{1}$, a subsequence converges almost everywhere in $\mathbb{R}^{3}$. In addition, the norms converge, $\left\|u_{n}\right\|_{L^{1}}=M=\left\|\chi_{\Omega^{0}}\right\|_{L^{1}}$, so by the Brezis-Lieb Lemma (see Theorem 1.9 in Lieb and Loss [14]) we may then conclude that (along a subsequence) $u_{n} \rightarrow w^{0}=\chi_{\Omega^{0}}$ in $L^{1}$ norm. By the lower semicontinuity of the perimeter (Proposition 4.29 in Maggi [19]) and of the interaction terms (Lemma 2.3 of Frank and Lieb [6])

$$
\int_{\mathbb{R}^{3}}\left|\nabla w^{0}\right| \leqslant \liminf _{n \rightarrow \infty} \int_{\mathbb{R}^{3}}\left|\nabla u_{n}\right| \quad \int_{\mathbb{R}^{3}} \int_{\mathbb{R}^{3}} \frac{w^{0}(x) w^{0}(y)}{|x-y|} d x d y \leqslant \liminf _{n \rightarrow \infty} \int_{\mathbb{R}^{3}} \int_{\mathbb{R}^{3}} \frac{u_{n}(x) u_{n}(y)}{|x-y|} d x d y .
$$

To pass to the limit in the confinement term, we apply Lemma 4 to the sequence $u_{n}-w^{0} \rightarrow$ 0 in $L^{1}\left(\mathbb{R}^{3}\right)$, and together with the above we have

$$
\mathrm{E}_{V}\left(w^{0}\right) \leqslant \liminf _{n \rightarrow \infty} \mathrm{E}_{V}\left(u_{n}\right) .
$$

Therefore we conclude that $w^{0}=\chi_{\Omega^{0}}$ attains the minimum value of $E_{V}$, and the proof is complete. To derive a contradiction, we now assume that $m_{0}:=\left|\Omega^{0}\right|<M$.

Step 2. Next, we show that the energy splits. First, assume that $0<\left|\Omega^{0}\right|<M$. We apply Lemma 2.2 of Frank and Lieb [6] (with $x_{n}=x_{n}^{0}=0$ ): there exists $r_{n}>0$ such that the sets

satisfy

$$
\mathcal{U}_{n}^{0}=\Omega_{n} \cap B_{r_{n}} \quad \text { and } \quad \mathcal{V}_{n}^{0}=\Omega_{n} \cap\left(\mathbb{R}^{3} \backslash \bar{B}_{r_{n}}\right)
$$

$$
\begin{gathered}
\chi_{\mathcal{U}_{n}^{0}} \rightarrow \chi_{\Omega^{0}} \quad \text { in } L^{1}\left(\mathbb{R}^{3}\right), \quad \chi_{\mathcal{V}_{n}^{0}} \rightarrow 0 \quad \text { in } L_{\text {loc }}^{1}\left(\mathbb{R}^{3}\right), \\
\lim _{n \rightarrow \infty}\left|\mathcal{U}_{n}^{0}\right|=\left|\Omega^{0}\right|=m_{0}, \quad \operatorname{Per} \Omega^{0} \leqslant \liminf _{n \rightarrow \infty} \operatorname{Per} \mathcal{U}_{n}^{0}, \\
\text { and } \quad \lim _{n \rightarrow \infty}\left(\operatorname{Per} \Omega_{n}-\operatorname{Per} \mathcal{U}_{n}^{0}-\operatorname{Per} \mathcal{V}_{n}^{0}\right)=0 .
\end{gathered}
$$

We now define $w_{n}^{0}(x):=\chi_{\mathcal{U}_{n}^{0}}(x), w^{0}(x):=\chi_{\Omega^{0}}(x), \Omega_{n}^{0}:=\mathcal{V}_{n}^{0}$, and $u_{n}^{0}(x):=\chi_{\Omega_{n}^{0}}(x)$ so that $u_{n}=w_{n}^{0}+u_{n}^{0}=w^{0}+u_{n}^{0}+o(1)$ in $L^{1}\left(\mathbb{R}^{3}\right)$, and $u_{n}^{0} \rightarrow 0$ in $L_{\text {loc }}^{1}$. In particular, by Lemma 4 .

$$
\int_{\mathbb{R}^{3}} V u_{n} d x=\int_{\mathbb{R}^{3}} V w^{0} d x+o(1) .
$$


Using Lemma 2.3 in Frank and Lieb [6], the nonlocal interaction term in $\mathrm{E}_{V}$ splits in a similar way as the perimeter,

$$
\begin{aligned}
\int_{\mathbb{R}^{3}} \int_{\mathbb{R}^{3}} \frac{u_{n}(x) u_{n}(y)}{|x-y|} d x d y & =\int_{\mathbb{R}^{3}} \int_{\mathbb{R}^{3}} \frac{w_{n}^{0}(x) w_{n}^{0}(y)}{|x-y|} d x d y+\int_{\mathbb{R}^{3}} \int_{\mathbb{R}^{3}} \frac{u_{n}^{0}(x) u_{n}^{0}(y)}{|x-y|} d x d y+o(1) \\
& =\int_{\mathbb{R}^{3}} \int_{\mathbb{R}^{3}} \frac{w^{0}(x) w^{0}(y)}{|x-y|} d x d y+\int_{\mathbb{R}^{3}} \int_{\mathbb{R}^{3}} \frac{u_{n}^{0}(x) u_{n}^{0}(y)}{|x-y|} d x d y+o(1),
\end{aligned}
$$

and thus the energy splits, up to a small error,

$$
\mathrm{E}_{V}\left(u_{n}\right)=\mathrm{E}_{V}\left(w_{n}^{0}\right)+\mathrm{E}_{0}\left(u_{n}^{0}\right)+o(1) \geqslant \mathrm{E}_{V}\left(w^{0}\right)+\mathrm{E}_{0}\left(u_{n}^{0}\right)+o(1) .
$$

In the case $\left|\Omega^{0}\right|=0$ (which we eliminate in Step 4 below, ) this splitting becomes trivial, with $w^{0} \equiv 0$ and $u_{n}^{0}=u_{n}$.

Step 3. Now we repeat the above procedure to locate a concentration set for the remainder $u_{n}^{0}$. We argue as above, but with $u_{n}^{0}$ replacing $u_{n}$, that is, the remainder set $\Omega_{n}^{0}=\mathcal{V}_{n}^{0}$ replacing $\Omega_{n}$. We know that $u_{n}^{0}=\chi_{\Omega_{n}^{0}} \rightarrow 0$ locally in $L^{1}\left(\mathbb{R}^{3}\right),\left|\Omega_{n}^{0}\right|=M-m_{0}+o(1) \in$ $(0, M]$, and $\mathrm{E}_{V}\left(u_{n}^{0}\right)$ (and hence $\left.\operatorname{Per} \Omega_{n}^{0}\right)$ are uniformly bounded. By Proposition 2.1 in Frank and Lieb [6] there exists a set $\Omega^{1}$ with $0<\left|\Omega^{1}\right| \leqslant M-m_{0}$ and a sequence of translations $x_{n} \in \mathbb{R}^{3}$ such that for some subsequence $\chi_{\Omega_{n}^{0}-x_{n}} \rightarrow \chi_{\Omega^{1}}$ in $L_{\text {loc }}^{1}\left(\mathbb{R}^{3}\right)$. Since $\chi_{\Omega_{n}^{0}} \rightarrow 0 L_{\text {loc }}^{1}\left(\mathbb{R}^{3}\right)$, we have that the translation points $\left|x_{n}\right| \rightarrow \infty$ as $n \rightarrow \infty$. Again, by Lemmas 2.2 and 2.3 of Frank and Lieb [6], and Lemma 4 as in Step 2, we similarly obtain a disjoint decomposition $\Omega_{n}^{0}-x_{n}=\mathcal{U}_{n}^{1} \cup \mathcal{V}_{n}^{1}$, with $\chi_{\mathcal{U}_{n}^{1}} \rightarrow \chi_{\Omega^{1}}$ in $L^{1}\left(\mathbb{R}^{3}\right), \chi_{\mathcal{V}_{n}^{1}} \rightarrow 0$ in $L_{\text {loc }}^{1}\left(\mathbb{R}^{3}\right)$, and for which the energy splits as in (2.1), namely,

$$
\mathrm{E}_{V}\left(u_{n}^{0}\right)=\mathrm{E}_{0}\left(u_{n}^{0}\right)+o(1) \geqslant \mathrm{E}_{0}\left(w^{1}\right)+\mathrm{E}_{0}\left(u_{n}^{1}\right)+o(1),
$$

where $w^{1}:=\chi_{\Omega^{1}}, u_{n}^{1}=\chi_{\mathcal{V}_{n}^{1}+x_{n}} \rightarrow 0$ in $L_{\text {loc }}^{1}\left(\mathbb{R}^{3}\right)$, and $\left|\mathcal{V}_{n}^{1}\right|=\left|\mathcal{V}_{n}^{0}\right|-m_{1}+o(1)$. We denote the re-centered remainder set $\Omega_{n}^{1}:=\mathcal{V}_{n}^{1}+x_{n}$, so that $u_{n}^{1}(x)=\chi_{\Omega_{n}^{1}}(x)$. Combining with the previous step, we now have

$$
\mathrm{E}_{V}\left(u_{n}\right) \geqslant \mathrm{E}_{V}\left(w^{0}\right)+\mathrm{E}_{0}\left(w^{1}\right)+\mathrm{E}_{0}\left(u_{n}^{1}\right)+o(1) \quad \text { and } \quad M=m_{0}+m_{1}+\left|\Omega_{n}^{1}\right|+o(1) .
$$

This, combined with the continuity of $e_{0}$ (see e.g. Lemma 4.8 in the work of Knüpfer, Muratov and Novaga [9]) yields a lower bound estimate in case of splitting,

$$
e_{V}(M) \geqslant e_{V}\left(m_{0}\right)+e_{0}\left(m_{1}\right)+e_{0}\left(M-m_{0}-m_{1}\right) \text {. }
$$

Step 4. We claim that $w^{0} \not \equiv 0$. For a contradiction, assume $w^{0} \equiv 0$. Define a sequence by $w_{n}(x):=u_{n}\left(x+x_{n}\right)$ using the translation sequence found above. Then $w_{n} \rightarrow w^{1}$ in $L^{1}\left(\mathbb{R}^{3}\right)$ and $w_{n}-u_{n}^{0} \rightarrow 0$ in $L_{\text {loc }}^{1}\left(\mathbb{R}^{3}\right)$. This implies, by Lemma 4, that $\lim _{n \rightarrow \infty} \int_{\mathbb{R}^{3}} V\left(w_{n}-u_{n}^{0}\right) d x=$ 0 . Now this limit and the translation invariance of the first two terms of $\mathrm{E}_{V}$ yield

$$
\mathrm{E}_{V}\left(w_{n}\right)-\mathrm{E}_{V}\left(u_{n}\right)=-\int_{\mathbb{R}^{3}} V\left(w_{n}-u_{n}\right) d x \longrightarrow-\int_{\mathbb{R}^{3}} V w^{1} d x<0
$$

hence, a contradiction.

Step 5. Now we prove that $e_{V}\left(m_{0}\right)=\mathrm{E}_{V}\left(w^{0}\right)$ and $e_{0}\left(m_{1}\right)=\mathrm{E}_{0}\left(w^{1}\right)$. By subadditivity (see Lemma 4 of Lu and Otto [17] and Lemma 3 in their earlier work [18], or Step 5 below) we have a rough upper bound estimate of the form

$$
e_{V}(M) \leqslant e_{V}\left(m_{0}\right)+e_{0}\left(m_{1}\right)+e_{0}\left(M-m_{0}-m_{1}\right)
$$


Combined with (2.2), this yields

$$
\begin{aligned}
e_{V}\left(m_{0}\right)+e_{0}\left(m_{1}\right)+e_{0}\left(M-m_{0}-m_{1}\right) & \geqslant e_{V}(M) \\
& \geqslant \mathrm{E}_{V}\left(w^{0}\right)+\mathrm{E}_{0}\left(w^{1}\right)+\liminf _{n \rightarrow \infty} \mathrm{E}_{0}\left(u_{n}^{1}\right) \\
& \geqslant e_{V}\left(m_{0}\right)+e_{0}\left(m_{1}\right)+e_{0}\left(M-m_{0}-m_{1}\right) .
\end{aligned}
$$

Hence,

$$
\left(\mathrm{E}_{V}\left(w^{0}\right)-e_{V}\left(m_{0}\right)\right)+\left(\mathrm{E}_{V}\left(w^{1}\right)-e_{V}\left(m_{1}\right)\right)+\left(\liminf _{n \rightarrow \infty} \mathrm{E}_{0}\left(u_{n}^{1}\right)-e_{0}\left(M-m_{0}-m_{1}\right)\right)=0,
$$

and since every term in this sum are nonnegative we must conclude that

$$
\mathrm{E}_{V}\left(w^{0}\right)=e_{V}\left(m_{0}\right) \quad \text { and } \quad \mathrm{E}_{0}\left(w^{1}\right)=e_{0}\left(m_{1}\right) .
$$

Step 6. Finally, we show, by means of an improved upper bound, that splitting leads to a contradiction, and hence the minimum must be attained. It is here that we use the superNewtonian attraction hypothesis (H2), Since $w^{0}=\chi_{\Omega^{0}}(x), w^{1}=\chi_{\Omega^{1}}(x)$ are minimizers of $e_{V}$ and $e_{0}$ respectively, by regularity of minimizers [10,17] we may choose $R>0$ for which $\Omega^{0}, \Omega^{1} \subset B_{R}(0)$. Let $b \in \mathbb{S}^{2}$ be any unit vector. For $t$ sufficiently large so that $\Omega^{0} \cap\left(\Omega^{1}+t b\right)=\emptyset$, let

$$
F(t):=\int_{\mathbb{R}^{3}} \int_{\mathbb{R}^{3}} \frac{w^{0}(x) w^{1}(y-t b)}{4 \pi|x-y|} d x d y, \text { and } G(t):=\int_{\mathbb{R}^{3}} V(x) w^{1}(x-t b) d x .
$$

We now estimate each; first,

$$
F(t) \leqslant \int_{B_{R}(0)} \int_{B_{R}(t b)} \frac{1}{4 \pi|x-y|} d x d y \leqslant \frac{\left|B_{R}\right|^{2}}{4 \pi(t-2 R)} \leqslant \frac{\left|B_{R}\right|^{2}}{2 \pi t},
$$

for all $t$ large enough.

To estimate $G(t)$ from below, we recall from (H2) that for any $A>0$ there exists $t_{1}>0$ such that for all $t>t_{1}$,

$$
\inf _{|x|=t} V(x) \geqslant \frac{A}{t}
$$

Thus, for each $i=1, \ldots, N$, as $t \rightarrow \infty$,

$$
t \int_{\mathbb{R}^{3}} V(x) w^{1}(x-t b) d x=\int_{\Omega^{1}} t V(x+t b) d x \geqslant \int_{\Omega^{1}} \frac{t A}{|x+t b|} d x \rightarrow A\left|\Omega^{1}\right|,
$$

by dominated convergence, and hence $\lim _{t \rightarrow \infty} t G(t)=\infty$. Thus, $t(F(t)-G(t)) \rightarrow-\infty$ as $t \rightarrow \infty$. Choose $\epsilon>0$ and $t_{0}>0$ such that

$$
F\left(t_{0}\right)-G\left(t_{0}\right)<-\epsilon<0 .
$$

With this choice of $\epsilon>0$, we may choose a compact set $K=K(\epsilon)$ for which $|K|=$ $M-m_{0}-m_{1}$ and

$$
\mathrm{E}_{0}\left(\chi_{K}\right)<e_{0}\left(M-m_{0}-m_{1}\right)+\frac{\epsilon}{3} .
$$

Choose $\tau>0$ large enough so that $K_{\tau}:=K-\tau b$ satifies

$$
\int_{\Omega^{i}} \int_{K_{\tau}} \frac{1}{4 \pi|x-y|} d x d y<\frac{\epsilon}{3}, \quad \text { for } \quad i=0,1
$$


Using $v(x)=w^{0}(x)+w^{1}\left(x-t_{0} b\right)+\chi_{K_{\tau}}$ as a test function, which is admissible for $e_{V}(M)$, we have

$$
\begin{aligned}
e_{V}(M) \leqslant \mathrm{E}_{V}(v)= & \mathrm{E}_{V}\left(w^{0}\right)+\mathrm{E}_{0}\left(w^{1}\right)+\mathrm{E}_{0}\left(\chi_{K_{\tau}}\right)+F\left(t_{0}\right)-G\left(t_{0}\right) \\
& +\sum_{i=0,1} \int_{\Omega^{i}} \int_{K_{\tau}} \frac{1}{4 \pi|x-y|} d x d y-\int_{K_{\tau}} V(x) d x \\
\leqslant & e_{V}\left(m_{0}\right)+e_{0}\left(m_{1}\right)+e_{0}\left(M-m_{0}-m_{1}\right)-\frac{\epsilon}{3},
\end{aligned}
$$

which contradicts the lower bound in case of splitting, (2.2). Thus we must have $\left|\Omega^{0}\right|=M$ and $e_{V}(M)=\mathrm{E}_{V}\left(w^{0}\right)$, for any $M>0$.

\section{Proof of Theorem 2}

Now we turn our attention to $\mathscr{E}_{V}$ and $I_{V}(M)$ given by (1.2) and (TFDW), respectively. As in the previous section, we define the "problem at infinity" by

$$
I_{0}(M):=\inf \left\{\mathscr{E}_{0}(u): u \in H^{1}\left(\mathbb{R}^{3}\right), \int_{\mathbb{R}^{3}}|u|^{2} d x=M\right\}
$$

where

$$
\mathscr{E}_{0}(u):=\int_{\mathbb{R}^{3}}\left(|\nabla u|^{2}+|u|^{10 / 3}-|u|^{8 / 3}\right) d x+\frac{1}{2} \int_{\mathbb{R}^{3}} \int_{\mathbb{R}^{3}} \frac{|u(x)|^{2}|u(y)|^{2}}{|x-y|} d x d y .
$$

First we note that the problems $I_{V}$ and $I_{0}$ satisfy the following "binding inequality", which is the standard subadditivity condition from concentration-compactness principle. For the proof of the following lemma we refer to Lemma 5 in Nam and van den Bosch [22].

Lemma 5. For all $0<m<M$ we have that

$$
I_{V}(M) \leqslant I_{V}(m)+I_{0}(M-m) .
$$

Moreover, $I_{V}(M)<I_{0}(M)<0, I_{V}(M)$ is continuous and strictly decreasing in $M$.

Next we prove that the ground-state value $I_{V}(M)$ is bounded.

Lemma 6. Let $\left\{u_{n}\right\}_{n \in \mathbb{N}} \subset H^{1}\left(\mathbb{R}^{3}\right)$ be a minimizing sequence for the energy $\mathscr{E}_{V}$ with $\int_{\mathbb{R}^{3}}\left|u_{n}\right|^{2} d x=M$. Then there exists constant $C_{0}>0$ such that $\left\|u_{n}\right\|_{H^{1}\left(\mathbb{R}^{3}\right)}^{2} \leqslant C_{0} M$.

Proof. First, we note that $I_{V}(M)<0$ for any $M>0$. Indeed, in the proof Lemma 5 of Nam and van den Bosch 22] it is shown that $I_{0}(M)<0$, and $\mathscr{E}_{V}(u) \leqslant \mathscr{E}_{0}(u)$ holds for all $u \in H^{1}\left(\mathbb{R}^{3}\right)$ with $\int_{\mathbb{R}^{3}}|u|^{2} d x=M$. We first claim that the quadratic form defined by the Schrödinger operator $-\Delta-V(x)$ is bounded below, i.e., that there exists $\lambda>0$ with

$$
\int_{\mathbb{R}^{3}}\left(|\nabla u|^{2}-V(x)|u|^{2}\right) d x \geqslant \frac{1}{2}\|u\|_{H^{1}}^{2}-\lambda\|u\|_{L^{2}}^{2},
$$

for all $u \in H^{1}\left(\mathbb{R}^{3}\right)$. To see this, we note that by $\left(\mathrm{H}^{\prime}\right)$ we may write $V=V_{1}+V_{2}$ with $V_{1} \in L^{3 / 2}\left(\mathbb{R}^{3}\right)$ and $V_{2} \in L^{\infty}\left(\mathbb{R}^{3}\right)$. Moreover, we may assume that $\left\|V_{1}\right\|_{L^{3 / 2}\left(\mathbb{R}^{3}\right)}<\epsilon$ for some $\epsilon>0$ to be chosen later. By the Hölder and Sobolev inequalities it follows that

$$
\int_{\mathbb{R}^{3}}\left|V_{1}\right||u|^{2} d x \leqslant\left\|V_{1}\right\|_{L^{3 / 2}\left(\mathbb{R}^{3}\right)}\|u\|_{L^{6}\left(\mathbb{R}^{3}\right)}^{2} \leqslant \epsilon S_{3}\|\nabla u\|_{L^{2}\left(\mathbb{R}^{3}\right)}^{2},
$$


where $S_{3}>0$ is the Sobolev constant. Thus,

$$
\int_{\mathbb{R}^{3}}\left(|\nabla u|^{2}-V(x)|u|^{2}\right) d x \geqslant\left(1-\epsilon S_{3}\right)\|\nabla u\|_{L^{2}\left(\mathbb{R}^{3}\right)}^{2}-\left\|V_{2}\right\|_{L^{\infty}\left(\mathbb{R}^{3}\right)}\|u\|_{L^{2}\left(\mathbb{R}^{3}\right)}^{2},
$$

and the lower bound is obtained by choosing

$$
\epsilon=\frac{1}{2 S_{3}} \quad \text { and } \quad \lambda=\left\|V_{2}\right\|_{L^{\infty}\left(\mathbb{R}^{3}\right)}+\frac{1}{2} .
$$

Using the elementary inequality

$$
|u|^{10 / 3}-|u|^{8 / 3}=\left(|u|^{5 / 3}-\frac{1}{2}|u|\right)^{2}-\frac{1}{4}|u|^{2} \geqslant-\frac{1}{4}|u|^{2}
$$

to estimate the nonlinear potential, we obtain the lower bound

$$
\begin{aligned}
\mathscr{E}_{V}\left(u_{n}\right) & \geqslant \int_{\mathbb{R}^{3}}\left(\left|\nabla u_{n}\right|^{2}-V(x)\left|u_{n}\right|^{2}\right) d x-\frac{1}{4}\left\|u_{n}\right\|_{L^{2}\left(\mathbb{R}^{3}\right)}^{2} \\
& \geqslant \frac{1}{2}\left\|u_{n}\right\|_{H^{1}}^{2}-\left(\lambda+\frac{1}{4}\right)\left\|u_{n}\right\|_{L^{2}\left(\mathbb{R}^{3}\right)}^{2}=\frac{1}{2}\left\|u_{n}\right\|_{H^{1}}^{2}-\frac{C_{0}}{2} M
\end{aligned}
$$

Since $I_{V}(M)<0$, for $n \in \mathbb{N}$ sufficiently large we have that $\mathscr{E}_{V}\left(u_{n}\right)<0$. Referring back to the above inequalities we obtain $\left\|u_{n}\right\|_{H^{1}\left(\mathbb{R}^{3}\right)}^{2} \leqslant C_{0} M$.

We now begin the proof of Theorem 2 .

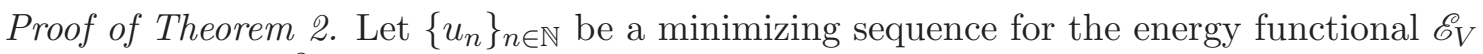
such that $\int_{\mathbb{R}^{3}}\left|u_{n}\right|^{2} d x=M$.

Step 1. First, note that by the uniform $H^{1}$-bound in Lemma 6 we may extract a subsequence so that $u_{n} \rightarrow v^{0}$ weakly in $H^{1}\left(\mathbb{R}^{3}\right)$ and strongly in $L_{\text {loc }}^{q}\left(\mathbb{R}^{3}\right)$ for all $2 \leqslant q<6$. Let $v_{n}:=u_{n}-v^{0}$, so $v_{n} \rightarrow 0$ weakly in $H^{1}\left(\mathbb{R}^{3}\right)$ and strongly in $L^{q}\left(\mathbb{R}^{3}\right)$ on compact sets as $n \rightarrow \infty$. In particular, by hypotheses $\left(\mathrm{H} 1^{\prime}\right)$, (H3) we have that

$$
\int_{\mathbb{R}^{3}} V(x)\left|v_{n}\right|^{2} d x \rightarrow 0
$$

as $n \rightarrow \infty$. Combining this with the arguments in equations (62)-(64) of Nam and van den Bosch [22] we may conclude that the energy $\mathscr{E}_{V}$ splits as

$$
\lim _{n \rightarrow \infty}\left(\mathscr{E}_{V}\left(u_{n}\right)-\mathscr{E}_{V}\left(v^{0}\right)-\mathscr{E}_{0}\left(v_{n}\right)\right)=0 .
$$

(Note that at this point it is possible that $v^{0}=0$, i.e., the first component is trivial, but later we will in fact show that $v^{0} \not \equiv 0$, and thus it is a ground-state of $\mathscr{E}_{V}$.) Define

$$
m_{0}:=\int_{\mathbb{R}^{3}}\left|v^{0}\right|^{2} d x \in[0, M]
$$

Note also that weak convergence implies $\left\|v_{n}\right\|_{L^{2}\left(\mathbb{R}^{3}\right)}^{2} \rightarrow M-m_{0}$. In case $m_{0}>0$, we observe that (3.2) also implies

$I_{V}(M)=\mathscr{E}_{V}\left(v^{0}\right)+\lim _{n \rightarrow \infty} \mathscr{E}_{0}\left(v_{n}\right) \geqslant I_{V}\left(m_{0}\right)+\lim _{n \rightarrow \infty} I_{0}\left(\left\|v_{n}\right\|_{L^{2}\left(\mathbb{R}^{3}\right)}^{2}\right)=I_{V}\left(m_{0}\right)+I_{0}\left(M-m_{0}\right)$,

by the continuity of $I_{0}$. As the result of Lemma 5 gives the opposite inequality, we conclude that

$$
I_{V}(M)=I_{V}\left(m_{0}\right)+I_{0}\left(M-m_{0}\right)
$$


In addition, $\mathscr{E}_{V}\left(v^{0}\right)=I_{V}\left(m_{0}\right)$; hence, $v^{0}$ is a ground-state, and $\left\{v_{n}\right\}_{n \in \mathbb{N}}$ is a minimizing sequence for $I_{0}\left(m_{1}\right)$ with $m_{1}=M-m_{0}$, i.e., $I_{0}\left(m_{1}\right)=\lim _{n \rightarrow \infty} \mathscr{E}_{0}\left(v_{n}\right)$.

If $m_{0}=M$ then the minimizing sequence is compact, and the proof is complete. Therefore, we will assume for the remainder of the proof that $m_{0}<M$.

Step 2. Concentration-compactness for $0 \leqslant m_{0}<M$ : there is a subsequence of $\left\{u_{n}\right\}$ (not relabeled), a sequence of points $\left\{y_{n}\right\} \subset \mathbb{R}^{3}$, constants $m_{i}>0$, and functions $v^{i} \in H^{1}\left(\mathbb{R}^{3}\right)$ for $i=0,1$ with

$$
\left.\begin{array}{c}
u_{n}-\left(v^{0}+v^{1}\left(\cdot-y_{n}\right)\right) \rightarrow 0 \quad \text { in } L^{2}\left(\mathbb{R}^{3}\right), \\
m_{0}+m_{1} \leqslant M, \quad \int_{\mathbb{R}^{3}}\left|v^{i}\right|^{2} d x=m_{i}, \quad \mathscr{E}_{V}\left(v^{0}\right)=I_{V}\left(m_{0}\right), \quad \mathscr{E}_{0}\left(v^{1}\right)=I_{0}\left(m_{1}\right), \\
\text { and } I_{V}(M)=I_{V}\left(m_{0}\right)+I_{0}\left(m_{1}\right)+I_{0}\left(M-m_{0}-m_{1}\right) .
\end{array}\right\}
$$

This concentration-compactness result is very similar to Steps 1-3 of the proof of Theorem 11, and in fact it follows immediately from steps (i) and (ii) of the proof of Lemma 9 of Nam and van den Bosch [22. (See also the Appendix of Lions [15].)

Step 3. Next, we claim that $v^{0} \not \equiv 0$. This follows by the same arguments as in Step 4 of the proof of Theorem 1. Indeed, assume the contrary, so $m_{0}=0$. Then by Lemma 5 and (3.3) we would have

$$
I_{V}(M) \leqslant I_{0}(M) \leqslant I_{0}\left(m_{1}\right)+I_{0}\left(M-m_{1}\right)=I_{V}(M),
$$

and so $I_{V}(M)=I_{0}(M)$. But the energy functional $\mathscr{E}_{0}$ is translation invariant, hence we may pull back the component, $\widetilde{u}_{n}(x):=u_{n}\left(x+y_{n}\right)$ with the same $\mathscr{E}_{0}$ value, and obtain

$$
\begin{aligned}
I_{V}(M) & =I_{0}(M)=\lim _{n \rightarrow \infty} \mathscr{E}_{0}\left(\widetilde{u}_{n}\right)=\lim _{n \rightarrow \infty}\left[\mathscr{E}_{V}\left(\widetilde{u}_{n}\right)+\int_{\mathbb{R}^{3}} V(x)\left|\widetilde{u}_{n}\right|^{2} d x\right] \\
& \geqslant I_{V}(M)+\liminf _{n \rightarrow \infty} \int_{\mathbb{R}^{3}} V(x)\left|\widetilde{u}_{n}\right|^{2} d x=I_{V}(M)+\int_{\mathbb{R}^{3}} V(x)\left|v^{1}\right|^{2} d x \\
& >I_{V}(M),
\end{aligned}
$$

a contradiction. Therefore $m_{0}>0$, and $v^{0}$ is a nontrivial ground-state of $I_{V}\left(m_{0}\right)$.

Step 4. Both $v^{0}$ and $v^{1}$ are strictly positive and have exponential decay, i.e., $0<v^{i}(x) \leqslant$ $C e^{-\nu|x|}$, for constants $C, \nu>0$ and for $i=0,1$. To show this, we first follow the Appendix in Lions [15] and note that, by Ekeland's variational principle [4], we may find a minimizing sequence $\tilde{u}_{n}$ for $I_{V}(M)$, with

$$
\left\|\tilde{u}_{n}-u_{n}\right\|_{H^{1}\left(\mathbb{R}^{3}\right)} \rightarrow 0
$$

and which approximately solve the Euler-Lagrange equations, $\left\|D \mathscr{E}_{V}\left(\tilde{u}_{n}\right)-\mu_{n} \tilde{u}_{n}\right\|_{H^{-1}\left(\mathbb{R}^{3}\right)} \rightarrow$ 0 , that is,

$$
-\Delta \tilde{u}_{n}+\left[f\left(\tilde{u}_{n}\right)-V(x)\left(\left|\tilde{u}_{n}\right|^{2} *|x|^{-1}\right)-\mu_{n}\right] \tilde{u}_{n} \longrightarrow 0,
$$

in $H^{-1}\left(\mathbb{R}^{3}\right)$, with $f(t)=\frac{5}{3} t^{4 / 3}-\frac{4}{3} t^{2 / 3}$, and Lagrange multiplier $\mu_{n}$. As $\left\|\tilde{u}_{n}\right\|_{H^{1}\left(\mathbb{R}^{3}\right)}$ is uniformly bounded, using $\tilde{u}_{n}$ as a test function we readily show that the Lagrange multipliers $\mu_{n}$ are bounded, and passing to a limit along a subsequence, $\mu_{n} \rightarrow \mu$. Furthermore, by Step 2 and (3.4), $\tilde{u}_{n}$ admits the same decomposition (3.3) into components $v^{i}$ as does $u_{n}$, 
and using weak convergence we obtain a limiting PDE for each component,

$$
\begin{gathered}
-\Delta v^{0}+\left[f\left(v^{0}\right)-V(x)+\left(\left(v^{0}\right)^{2} *|x|^{-1}\right)\right] v^{0}=\mu v^{0}, \\
-\Delta v^{1}+\left[f\left(v^{1}\right)+\left(\left(v^{1}\right)^{2} *|x|^{-1}\right)\right] v^{1}=\mu v^{1},
\end{gathered}
$$

with the same Lagrange multiplier $\mu$. By minimization, $v^{i} \geqslant 0$ and by the strong maximum principle, we may conclude that each $v^{i}>0$ for $i=0,1$.

Next, we show that the Lagrange multiplier $\mu<0$. Following the proof of Theorem 1 of Le Bris [11], we define the spherical mean of an integrable $\psi$ as $\bar{\psi}(x)=$ $\frac{1}{4 \pi} \int_{\sigma \in \mathbb{S}^{2}} \psi(|x| \sigma) d S(\sigma)$, and note that by Newton's Theorem (see Theorem 9.7 of Lieb and Loss [14]),

$$
\overline{\left(v^{0}\right)^{2} *|x|^{-1}}=\overline{\left(v^{0}\right)^{2}} *|x|^{-1} \leqslant|x|^{-1} \int_{\mathbb{R}^{3}}\left(v^{0}\right)^{2} d x \leqslant \frac{M}{|x|} .
$$

By (H2), there exists $R>0$ for which $\bar{V}(x) \geqslant \frac{M}{|x|}$ for all $|x| \geqslant R$, and hence

$$
\overline{\left(v^{0}\right)^{2} *|x|^{-1}-V} \leqslant 0 \text { for all }|x| \geqslant R .
$$

Assume for a contradiction that $\mu \geqslant 0$. Set $W:=\frac{5}{3}\left|v^{0}\right|^{4 / 3}+\left[\left(v^{0}\right)^{2} *|x|^{-1}\right]-V$, so $v^{0}$ satisfies the differential inequality,

$$
-\Delta v^{0}+W v^{0}=\left(\frac{2}{3}\left(v^{0}\right)^{5 / 3}+\mu\right) v^{0} \geqslant 0
$$

in $\mathbb{R}^{3}$. By (3.5) , outside $B_{R}, \bar{W}_{+}=\frac{5}{3} \overline{\left(v^{0}\right)^{4 / 3}} \in L^{3 / 2}$. Applying Theorem 7.18 of Lieb [13], we conclude that $v^{0} \notin L^{2}\left(B_{R}^{c}\right)$, a contradiction. Thus, $\mu<0$.

Finally, from equation (66) of Lions [15] we may conclude that the solutions are exponentially localized,

$$
\left|\nabla v^{i}(x)\right|+\left|v^{i}(x)\right| \leqslant C e^{-\nu|x|}
$$

for $i=0,1$ with $0<\nu<\sqrt{-\mu}$.

Step 5. We are ready to complete the existence argument. Assume, for a contradiction, that $u_{n}$ is a minimizing sequence for $I_{V}(M)$ with no convergent subsequence. By Step 2, we obtain $m_{i}>0, v^{i} \in H^{1}\left(\mathbb{R}^{3}\right)$ for $i=0,1$ satisfying (3.3). Moreover, we claim that

$$
I_{V}\left(m_{0}+m_{1}\right)<I_{V}\left(m_{0}\right)+I_{0}\left(m_{1}\right) .
$$

Assuming the claim holds, taking $m=m_{0}+m_{1}$ in Lemma 5, and using (3.3), we obtain that

$$
\begin{aligned}
I_{V}(M) & \leqslant I_{V}\left(m_{0}+m_{1}\right)+I_{0}\left(M-m_{0}-m_{1}\right) \\
& <I_{V}\left(m_{0}\right)+I_{0}\left(m_{1}\right)+I_{0}\left(M-m_{0}-m_{1}\right) \\
& =I_{V}(M),
\end{aligned}
$$

a contradiction. We therefore conclude that $m_{0}=M$, and the minimizing sequence converges.

In order to prove (3.7) we will construct a family of functions based on the elements obtained in (3.3): For $t>0$, let

$$
w_{t}(x):=v^{0}(x)+v^{1}(x-t \xi),
$$


where $\xi \in \mathbb{R}^{3}$ with $|\xi|=1$, and define the admissible function

$$
\widetilde{w}_{t}(x):=\frac{\sqrt{m_{0}+m_{1}} w_{t}(x)}{\left\|w_{t}\right\|_{L^{2}\left(\mathbb{R}^{3}\right)}}
$$

so that $\int_{\mathbb{R}^{3}} \widetilde{w}_{t}^{2} d x=m_{0}+m_{1}$. However, by the exponential decay (3.6) we note that

$$
\left|\mathscr{E}_{V}\left(\widetilde{w}_{t}\right)-\mathscr{E}_{V}\left(w_{t}\right)\right| \leqslant C e^{-\nu t},
$$

and hence in order to estimate $\mathscr{E}_{V}\left(\widetilde{w}_{t}\right)$ it suffices to estimate $\mathscr{E}_{V}\left(w_{t}\right)$.

Again using the exponential decay of the component functions $v^{i}, i=0,1$, and arguing as in the proof of Corollary II.2(ii) in Lions [15], for $t>0$ large, we obtain the decomposition

$$
\begin{aligned}
\mathscr{E}_{V}\left(w_{t}\right)=\mathscr{E}_{V}\left(v^{0}\right)+I_{0}\left(m_{i}\right) \\
\quad+2 \int_{\mathbb{R}^{3}} \int_{\mathbb{R}^{3}} \frac{\left|v^{0}(x)\right|^{2}\left|v^{1}(y-t \xi)\right|^{2}}{4 \pi|x-y|} d x d y-\int_{\mathbb{R}^{3}} V(x)\left|v^{1}(x-t \xi)\right|^{2} d x+o\left(\frac{1}{t}\right) .
\end{aligned}
$$

Now we show that for large $t>0$, the second line above is strictly negative. First, note that

$$
\begin{aligned}
t \int_{\mathbb{R}^{3}} \int_{\mathbb{R}^{3}} \frac{\left|v^{0}(x)\right|^{2}\left|v^{1}(y-t \xi)\right|^{2}}{4 \pi|x-y|} d x d y=\frac{1}{4 \pi} \int_{\mathbb{R}^{3}} \int_{\mathbb{R}^{3}} \frac{\left|v^{0}(x)\right|^{2}\left|v^{1}(y)\right|^{2}}{|\xi-(x-y) / t|} d x d y \\
\qquad \underset{t \rightarrow \infty}{\longrightarrow} \frac{\left\|v^{0}\right\|_{L^{2}\left(\mathbb{R}^{3}\right)}^{2}\left\|v^{1}\right\|_{L^{2}\left(\mathbb{R}^{3}\right)}^{2}}{4 \pi|\xi|}=\frac{m_{i} m_{j}}{4 \pi}
\end{aligned}
$$

by dominated convergence theorem. That is, this term is $O\left(t^{-1}\right)$.

To estimate the other term, first note that (H2) implies that for every $A>0$ there exists $t_{0}>0$ such that $t V(x) \geqslant A$ for $|x|=t$ whenever $t \geqslant t_{0}$, i.e.,

$$
\inf _{|x|=t} V(x) \geqslant \frac{A}{|x|}
$$

when $|x|=t \geqslant t_{0}$. Next, choose $r_{0}$ and $C>0$ such that $\int_{B_{r_{0}}(0)}\left|v^{1}\right|^{2} d x \geqslant C>0$. Then, for $t>2 r_{0}$ we have that

$$
\begin{aligned}
t \int_{\mathbb{R}^{3}} V(x)\left|v^{1}(x-t \xi)\right|^{2} d x & \geqslant t \int_{B_{r_{0}}(0)} V(x+t \xi)\left|v^{1}(x)\right|^{2} d x \\
& \geqslant C t \inf _{x \in B_{r_{0}}(0)} V(x+t \xi) \\
& \geqslant C t \inf _{t-r_{0} \leqslant|x| \leqslant t+r_{0}} \frac{A}{|x|}=\frac{C t A}{t+r_{0}} \geqslant \frac{C A}{2}
\end{aligned}
$$

for large enough $t>0$. Since the above holds for all $A>0$ we have that

$$
t \int_{\mathbb{R}^{3}} V(x)\left|v^{1}(x-t \xi)\right|^{2} d x \underset{t \rightarrow \infty}{\longrightarrow} \infty .
$$

In particular, the confinement term dominates the other cross terms for $t>0$ sufficiently large, and thus

$$
I_{V}\left(m_{0}+m_{1}\right) \leqslant \mathscr{E}_{V}\left(\widetilde{w}_{t}\right)<I_{V}\left(m_{0}\right)+I_{0}\left(m_{1}\right),
$$

proving our claim (3.7). 
Acknowledgments. The authors would like to thank the referees for their invaluable comments, which allowed us to simplify the proof of Theorem 2 significantly. SA, LB, and RC were supported by NSERC (Canada) Discovery Grants.

\section{REFERENCES}

[1] J. D. Bekenstein, "Relativistic gravitation theory for the modified newtonian dynamics paradigm," Phys. Rev. D, vol. 70, p. 083509, 2004.

[2] R. Benguria, H. Brézis, and E. H. Lieb, "The Thomas-Fermi-von Weizsäcker theory of atoms and molecules," Comm. Math. Phys., vol. 79, no. 2, pp. 167-180, 1981.

[3] D. V. Bugg, "Mond-a review," Can. J. Phys., vol. 93, no. 2, pp. 119-125, 2015.

[4] I. Ekeland, "Nonconvex minimization problems," Bull. Amer. Math. Soc. (N.S.), vol. 1, no. 3, pp. 443-474, 1979.

[5] A. Finzi, "On the validity of Newton's law at a long distance," Monthly Notices Roy. Astronom. Soc., vol. 127, pp. 21-30, 1963.

[6] R. L. Frank and E. H. Lieb, "A compactness lemma and its application to the existence of minimizers for the liquid drop model," SIAM J. Math. Anal., vol. 47, no. 6, pp. 4436-4450, 2015.

[7] R. L. Frank, P. T. Nam, and H. van den Bosch, "The ionization conjecture in Thomas-Fermi-Dirac-von Weizsäcker theory," arXiv preprint arXiv:1606.07355, 2016.

[8] G. Gamow, "Mass defect curve and nuclear constitution," Proc. R. Soc. Lond. A, vol. 126, no. 803, pp. 632-644, 1930.

[9] H. Knüpfer, C. B. Muratov, and M. Novaga, "Low density phases in a uniformly charged liquid," Comm. Math. Phys., vol. 345, no. 1, pp. 141-183, 2016.

[10] H. Knüpfer and C. B. Muratov, "On an isoperimetric problem with a competing nonlocal term II: The general case," Comm. Pure Appl. Math., vol. 67, no. 12, pp. 1974-1994, 2014.

[11] C. Le Bris, "Some results on the Thomas-Fermi-Dirac-von Weizsäcker model," Differential Integral Equations, vol. 6, no. 2, pp. 337-353, 1993.

[12] C. Le Bris and P.-L. Lions, "From atoms to crystals: a mathematical journey," Bull. Amer. Math. Soc. (N.S.), vol. 42, no. 3, pp. 291-363, 2005.

[13] E. H. Lieb, "Thomas-Fermi and related theories of atoms and molecules," Rev. Modern Phys., vol. 53, no. 4, pp. 603-641, 1981.

[14] E. H. Lieb and M. Loss, Analysis, ser. Graduate Studies in Mathematics. American Mathematical Society, Providence, RI, 1997, vol. 14.

[15] P.-L. Lions, "Solutions of Hartree-Fock equations for Coulomb systems," Comm. Math. Phys., vol. 109, no. 1, pp. 33-97, 1987.

[16] — "The concentration-compactness principle in the calculus of variations. The locally compact case. I," Ann. Inst. H. Poincaré Anal. Non Linéaire, vol. 1, no. 2, pp. 109-145, 1984.

[17] J. Lu and F. Otto, "An isoperimetric problem with Coulomb repulsion and attraction to a background nucleus," arXiv preprint arXiv:1508.07172, 2015.

[18] — "Nonexistence of a minimizer for Thomas-Fermi-Dirac-von Weizsäcker model," Comm. Pure Appl. Math., vol. 67, no. 10, pp. 1605-1617, 2014.

[19] F. Maggi, Sets of Finite Perimeter and Geometric Variational Problems, 1st ed., ser. Cambridge Studies in Advanced Mathematics. Cambridge: Cambridge University Press, 2012, vol. 135.

[20] M. Milgrom, "A modification of the newtonian dynamics as a possible alternative to the hidden mass hypothesis," The Astrophysical Journal, vol. 270, pp. 365-370, 1983.

[21] —-, "Mond theory," Can. J. Phys., vol. 93, no. 2, pp. 107-118, 2015.

[22] P. T. Nam and H. van den Bosch, "Nonexistence in Thomas-Fermi-Dirac-von Weizsäcker theory with small nuclear charges," Math. Phys. Anal. Geom., vol. 20, no. 2, pp. Art. 6, 32, 2017.

[23] C. F. von Weizsäcker, "Zur Theorie der Kernmassen," Z. Phys. A, vol. 96, no. 7, pp. 431-458, 1935. 
Dept of Math and Stat, McMaster University, Hamilton, ON

E-mail address: alama@mcmaster.ca

Dept of Math and Stat, Mcmaster University, Hamilton, ON

E-mail address: bronsard@mcmaster.ca

Dept of Math and Stat, McGill University, Montréal, QC

E-mail address: rustum.choksi@mcgill.ca

Dept of Math and Appl Math, Virginia Commonwealth University, Richmond, VA

E-mail address: iatopaloglu@vcu.edu 